\title{
POINT-LOADED DISCS AND BLOCKS APPLICABLE TO TENSILE TESTING OF BRITTLE MATERIALS
}

\author{
F. J. APPL School of Aerospace, Mechanical, and Nuclear Engineering, University of Oklahoma
}

\begin{abstract}
A method of numerically approximating the solutions of plane-stress or plane-strain elasticity problems with boundary conditions consisting of concentrated forces or distributed loads is presented herein. The effect of each concentrated force (commonly termed a point load) that acts on the boundary is represented by a Flamant solution. Usually, the combined effect of these Flamant solutions indicates the presence of distributed loadings or 'residual stresses' on some portions of the boundary that are not consistent with the actual boundary conditions. The negatives of these 'residual stresses' are used as stress boundary conditions in a singular integral method of numerical analysis that is applicable to plane elasticity problems involving distributed loadings on the boundaries. Since the method presented herein involves only stress boundary conditions, the solutions are valid for both plane stress and plane strain.

The accuracy of this superposition method is demonstrated by consideration of a circular disc or cylinder subjected to diametrically opposed concentrated forces for which accuracy to within 0.2 per cent of the exact solution is obtained. Parametric analyses of rectangular and elliptical compression members subjected to point loads are presented. Results determined herein are found to compare relatively well with those determined in previous numerical and experimental investigations of specific cases. These results make possible the design and analysis of compression members used to evaluate the tensile fracture strength of brittle materials.
\end{abstract}

\section{INTRODUCTION}

PLANE-STRESS or plane-strain elasticity solutions for members subjected to concentrated forces at various locations on the boundary are often determined by superposition of elasticity solutions which predict the effect of the concentrated forces and other solutions such that the stress boundary conditions for the member are satisfied. The effect of a concentrated force (commonly termed a point load) which acts on the boundary can be represented by a Flamant solution $(\mathbf{I})^{\star}$. The superposition of a Flamant solution for each point load that acts on the boundary usually results in the prediction of 'residual stresses' on some portions of the boundary that are, in reality, stressfree. An admissible solution must then be superimposed to form a composite solution such that the 'residual stresses' are cancelled and the stress boundary conditions of the problem are satisfied. For example, the complete solution for the circular disc or cylinder subjected to two diametrically opposed concentrated forces consists of the sum of the effects of two Flamant solutions and a hydrostatic tensile stress, as is shown in detail in (I). An admissible function that will result in a superposed solution that satisfies the boundary conditions of the problem at hand is generally very complex and usually cannot be determined exactly.

Recently, Appl and Koerner (2) presented a singular integral method of numerical analysis that is applicable to plane elasticity problems having stress boundary conditions. As presented in (2), the method is applicable to those problems involving distributed loads on the boundary.

A method of numerically approximating the solutions of plane elasticity problems with stress boundary conditions

The MS. of this paper was received at the Institution of Mechanical Engineers on 8th fune 1971 and accepted for publication on 28th fanuary 1972. 32

* References are given in the Appendix. consisting of point loads and/or distributed loads is developed herein. Since the method presented involves only stress boundary conditions, the solutions are valid for either plane stress or plane strain. Each point load that acts on the boundary can be represented by a Flamant solution (I). The combined effect of these Flamant solutions will, in general, indicate the presence of distributed loadings or 'residual stresses' on other portions of the boundary that are not consistent with the actual boundary conditions. The negatives of these 'residual stresses' can then be used as stress boundary conditions in the singular integral method (2). The superposition of several Flamant solutions and the results of the singular integral method of numerical analysis give an approximate solution of plane elasticity problems with point loads acting on the boundary. This superposition method is exact in that the point loads acting on the boundary are represented by Flamant solutions. The superposition method is approximate in that the singular integral method of numerical analysis is a boundary-collocation method which satisfies the boundary conditions only at discrete points on the boundary. Thus the superposition method exactly satisfies the boundary conditions at the points of application of point loads and satisfies the boundary conditions on the remaining portions of the boundary at discrete points.

It is generally known that greater maximum tensile stresses for a given compressive load can be produced in non-circular blocks subjected to point loads than are produced in the diametrically loaded circular disc. Various geometrical configurations have been considered analytically by Goodier (3), Niedenfuhr, Leissa, and Lo (4), Gaydon (5), and Brisbane (6). The energy method used in (4) was so laborious that only three rectangular blocks were investigated. Niedenfuhr, Leissa, and Lo (4) used a pointmatching technique to determine the stress distributions in a square block loaded by compressive point loads and 
arrived at approximately the same results as in (3). Gaydon (5) used a series-solution boundary-collocation method in determining an approximate solution for a rectangular block with a width/height ratio of 2 . Excellent agreement between Gaydon's results and the present calculations is shown below. Using the complex-variable method, Brisbane (6) determined an infinite-series solution for the general problem of an elliptical disc subjected to diametrically opposed point loads.

The method developed herein was programmed for a digital computer and used to determine parametric analyses of rectangular compression blocks and elliptical discs. The results of these parametric analyses are found to compare well with specific cases previously presented and to determine more completely the effect of the geometrical parameters on the stress distributions in the various members.

\subsection{Notation}

$2 a$
$2 b$
$\mathrm{C}$
$\mathrm{M}$
$N$
$P$

Width of member (see Fig. 2).

Height of member (see Fig. 2).

Contour of member.

Point on the contour C.

Number of boundary segments used to approximate the contour $\mathrm{C}$.

Magnitude of an actual point load, force per unit depth.

$p_{1 i}, p_{2 i} \quad$ Magnitudes of the step density functions for the $i$ th boundary segment.

$p_{1}(M), p_{2}(M) \quad$ Magnitudes of the step density functions at point $M$.

Q Point inside contour C.

$R_{i} \quad$ Distance from the centre of the $i$ th boundary segment to point $Q$ (or $M$ ) (see Fig. 1).

$r \quad$ Distance from point of application of point load $P$ to point $M$ (or $Q$ ).

$S_{i} \quad$ Length of the $i$ th boundary segment (see Fig. 1).

$t$ Distance along boundary segment $S_{i}$, $-S_{i} / 2 \leqslant t \leqslant S_{i} / 2$.

$x, y \quad$ Rectangular co-ordinate axes.

$\alpha \quad$ Angle between $r$ and the direction of $P$.

$\theta_{i} \quad$ Angle from $x$ axis to $R_{i}$ (see Fig. 1).

$\nu \quad$ Poisson's ratio.

$\sigma_{n n}{ }^{T}(\mathrm{M}), \tau_{n t}{ }^{T}(\mathrm{M})$ Actual normal and shear stresses at point $\mathrm{M}$ (or $\mathrm{Q}$ ).

$\sigma_{n n}{ }^{1}(M), \tau_{n t}{ }^{1}(M)$ Normal- and shear-stress components at point $M$ (or $Q$ ) due to fictitious distributed loads, see equations (1) and (2) or (3) and (4).

$\sigma_{n n}^{2}(M), \tau_{n t}^{2}(M)$ Normal- and shear-stress components at point $M$ (or $Q$ ) due to actual point loads, see equations (5) and (6).

$\sigma_{n 1}, \tau_{n t 1} \quad$ Normal and shear stresses at point $M$ (or $\mathrm{Q}$ ) due to a fictitious point load acting in the $x$ direction at point $t$ in segment $S_{i}$.

$\sigma_{n 2}, \tau_{n t 2}$

Normal and shear stresses at point $M$ (or $\mathrm{Q}$ ) due to a fictitious load acting in the $y$ direction at point $t$ in segment $S_{i}$.

Angle from $r$ to direction $n$ at point $M$ (or Q).
$\phi_{t}$
Angle from $R_{i}$ to direction $n$ at point $M$ (or Q) (see Fig. 1). directed normal of boundary segment $S_{i}$ (see Fig. 1).
$\psi_{i} \quad$ Angle from $x$ axis to the outward
$\psi_{\mathbf{M}}$
Angle from the $x$ axis to the outward directed normal of the contour at point $M$.

\section{METHOD OF ANALYSIS}

The boundary or contour of the member of interest is approximated by $N$ straight-line segments. This contour $C$ is assumed to be scribed in the interior of an infinite plate. Each line segment is loaded by a fictitious distributed loading which has an arbitrary magnitude and is directed in the $x$ direction and by a similar fictitious loading in the $y$ direction. Each of these fictitious distributed loadings causes an effect at other points in the infinite plate. The effect at a point $M$ on the contour $C$ can be expressed as:

$$
\begin{aligned}
\sigma_{n n}{ }^{1}(\mathrm{M}) & =\frac{2 \pi}{(1-v)}\left[p_{1}(\mathrm{M}) \cos \psi_{\mathrm{M}}+p_{2}(\mathrm{M}) \sin \psi_{\mathrm{M}}\right] \\
& +\sum_{i=1}^{N}\left[p_{1 i} \int_{-S_{t} / 2}^{s_{i} / 2} \sigma_{n 1} \mathrm{~d} t+p_{2 i} \int_{-S_{i} / 2}^{s_{i} / 2} \sigma_{n 2} \mathrm{~d} t\right] \\
\tau_{n t}{ }^{1}(\mathrm{M}) & =\frac{2 \pi}{(1-\nu)}\left[-p_{1}(\mathrm{M}) \sin \psi_{\mathrm{M}}+p_{2}(\mathrm{M}) \cos \psi_{\mathrm{M}}\right] \\
& +\sum_{i=1}^{N}\left[p_{1 i} \int_{-S_{i} / 2}^{s_{i} / 2} \tau_{n t 1} \mathrm{~d} t+p_{2 i} \int_{-S_{i} / 2}^{S_{i} / 2} \tau_{n t 2} \mathrm{~d} t\right]
\end{aligned}
$$

or for a point $\mathrm{Q}$ inside the contour as:

$$
\begin{aligned}
& \sigma_{n n}{ }^{1}(\mathrm{Q})=\sum_{i=1}^{N}\left[p_{1 i} \int_{-S_{t} / 2}^{S_{i} / 2} \sigma_{n 1} \mathrm{~d} t+p_{2 i} \int_{-S_{t} / 2}^{S_{i} / 2} \sigma_{n 2} \mathrm{~d} t\right] \\
& \tau_{n t}{ }^{1}(\mathrm{Q})=\sum_{i=1}^{N}\left[p_{1 i} \int_{-S_{i} / 2}^{S_{i} / 2} \tau_{n t 1} \mathrm{~d} t+p_{2 i} \int_{-S_{i} / 2}^{S_{i} / 2} \tau_{n t 2} \mathrm{~d} t\right]
\end{aligned}
$$

$\sigma_{n 1}, \sigma_{n 2}, \tau_{n t 1}$, and $\tau_{n t 2}$ have been developed from Airy stress functions and represent the effects of fictitious point loads acting in the $x$ and $y$ directions. These expressions are presented in detail in (2).

The effect at point $M$ due to an actual point load acting on the boundary of the region of interest is represented by a Flamant solution (I) as

$$
\begin{gathered}
\sigma_{n n}^{2}(M)=-\frac{2 P \cos \alpha \cos ^{2} \phi}{\pi r} \\
\tau_{n t}{ }^{2}(M)=\frac{P \cos \alpha \sin 2 \phi}{\pi r}
\end{gathered}
$$

The actual stress boundary conditions at point $M$ (or $Q$ ) can be expressed as the sum of the effects at point $M$ (or $Q$ ) of the fictitious distributed loads and of the actual external point loads. That is:

$$
\begin{aligned}
& \sigma_{n n}{ }^{T}(M)=\sigma_{n n}{ }^{1}(M)+\sum \sigma_{n n}{ }^{2}(M) . \\
& \tau_{n t}{ }^{T}(M)=\tau_{n t}{ }^{1}(M)+\sum \tau_{n t}{ }^{2}(M) .
\end{aligned}
$$

where the summation in equations (7) and (8) indicates that the effect of each external point load is included.

The terms outside the summation sign on the righthand side of equations (1) and (2) represent the discontinuities in stress caused by the application of a fictitious distributed load along the boundary segment containing 
point $M$. This discontinuity is not present when an interior point $Q$ is considered, as shown by equations ( 3 ) and (4).

Defining the line from the centre of segment $S_{i}$ to point $\mathrm{Q}$ as $R_{i}$, the angle from the $x$ axis to the outward directed normal of segment $S_{i}$ as $\psi_{i}$, the angle from the $x$ axis to $R_{i}$ as $\theta_{i}$, and the angle from $R_{i}$ to direction $n$ at $\mathrm{Q}$ (or $M$ ) as $\phi_{i}$, we can express the results of the integrations indicated in equations (1)-(4) in terms of $R_{i}, \theta_{i}, \psi_{i}$, and $\phi_{i}$ for the general segment $S_{t}$. These geometrical variables are illustrated in Fig. 1. The solutions of the integrals in equations (1)-(4) have been presented in (2).

In the exact formulation of the problem, the summation signs are replaced by integrals over the contour in equations (1)-(4). Massonnet (7) has suggested iteration of the exact singular integral equations as a method of obtaining a solution. Lo, Niedenfuhr, and Leissa (8) approximated the continuous density function by a-step density function in their development of a singular integral method applicable to torsion and plate problems. By approximating the density function by a step function, the integrals over the contour can be replaced by summations of integrals over line segments. Appl and Koerner (2) used a similar manner of approximating the two continuous density functions by step functions in their work on plane elasticity problems.

In order to apply the superposition method to a specific problem, the boundary is first approximated by $N$ straightline segments. The actual stress boundary conditions are prescribed at the centre of each of the $N$ segments. These boundary conditions are the $\sigma_{n n}{ }^{T}(M)$ and $\tau_{n t}{ }^{T}(M)$ of a set of $2 \mathrm{~N}$ simultaneous equations arising from the use of equations (7) and (8) at each of the $N$ points. Once the $N$ segments have been defined, the integrals indicated in

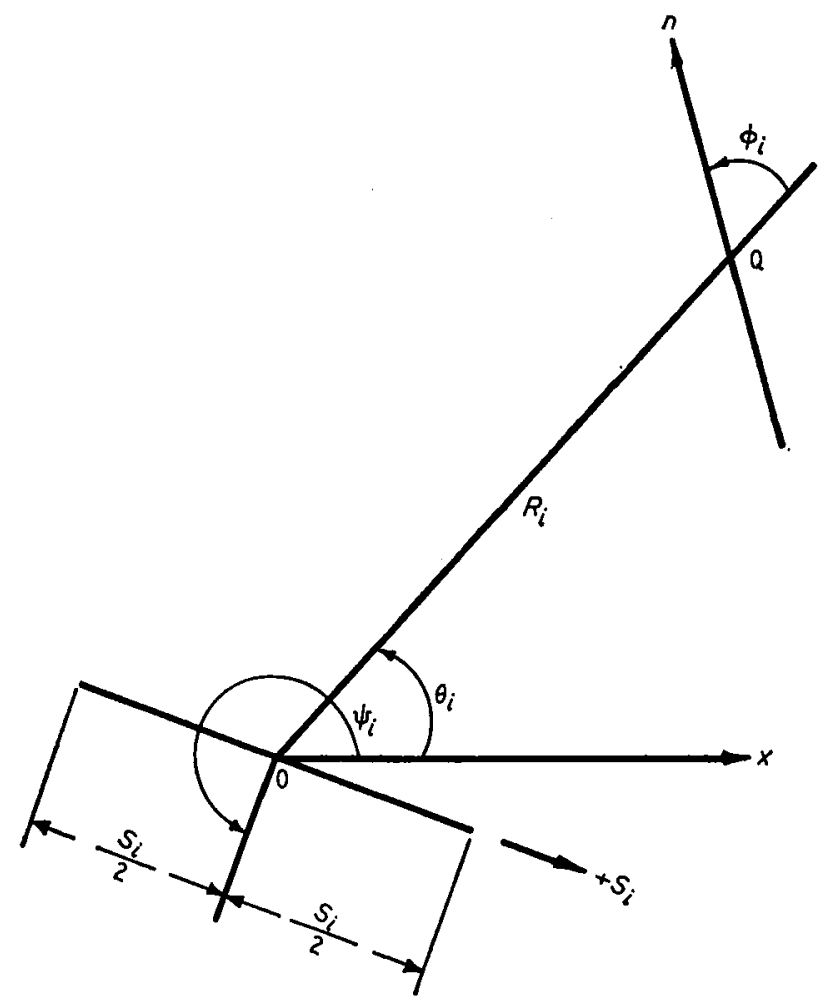

Fig. 1. Definition of co-ordinate system and geometrical variables for a general boundary segment equations (1) and (2) can be evaluated and are coefficients of the step density functions. The step density functions $p_{1 i}, p_{2 i}$ are the only unknown quantities in the set of $2 N$ simultaneous equations. After the step density functions have been evaluated, the stresses at other points and orientations on or interior to the contour can be evaluated by using equations (1) and (2) or (3) and (4) respectively in equations (7) and (8).

It should be noted that equations (1)-(4) contain singularity points which occur at the ends of the boundary segments $S_{i}$. However, the equations are well behaved at the mid-points of the segments and at all points inside the contour C. If all stress calculations for points on the boundary are made at the mid-points of the boundary segments, no difficulties are encountered.

This singular integral superposition method of numerical approximation is ideal for computer application because of the large number of repetitive calculations which must be made. A computer programme has been developed which takes as input the geometry, boundary conditions, and the locations where the stresses are desired. From the geometry and boundary conditions, coefficients of the set of simultaneous equations are computed by use of equations (1) and (2) in equations (7) and (8). This set of equations is solved for the magnitudes of the step density functions by inverting the matrix of coefficients. To find the stresses at specified locations, these step density functions are substituted back into equations (7) and (8). Equations (1) and (2) are used for points on the boundary and equations (3) and (4) for interior points.

\section{ANALYSIS OF RESULTS}

The singular integral superposition method of numerical analysis developed herein is used to make parametric analyses of the stress distributions in rectangular and elliptical members subjected to compressive point loads. The various geometrical shapes and the parameters used to describe these shapes and the loadings considered are shown in Fig. 2. All solutions were found to be independent of Poisson's ratio.

The stress distribution in a circular disc or cylinder subjected to diametrically opposed compressive concentrated forces was calculated in order to obtain a direct comparison between the results of the numerical analysis and the exact solution (I). For the determination of the numerical solution 192 segments were used to describe the boundary. The results were relatively insensitive to the placement of boundary points. To determine the effect of the number of segments, 160 were used for the solution of this problem. Since these two numerical analyses differed by less than 1 per cent it was concluded that 192 segments adequately define the boundary and that accurate results are obtained. As shown in Fig. 2, the diameter of the disc is $2 b$ and the external compressive point load per unit depth is $P$. The $x-y$ co-ordinate system is defined in Fig. 2 with its origin at the centre of the disc. The exact solution (I) predicts a uniform tensile stress in the $x$ direction at all points along the $y$ axis and a compressive-stress distribution in the $y$ direction at points along the $x$ axis that is a maximum at the origin and decreases to zero at the outer boundary. The exact solution is plotted as the solid lines in Fig. 3 while the data points represent the results of the numerical analysis. The maximum difference between the exact solution and the results of the numerical analysis is 0.2 per cent. 

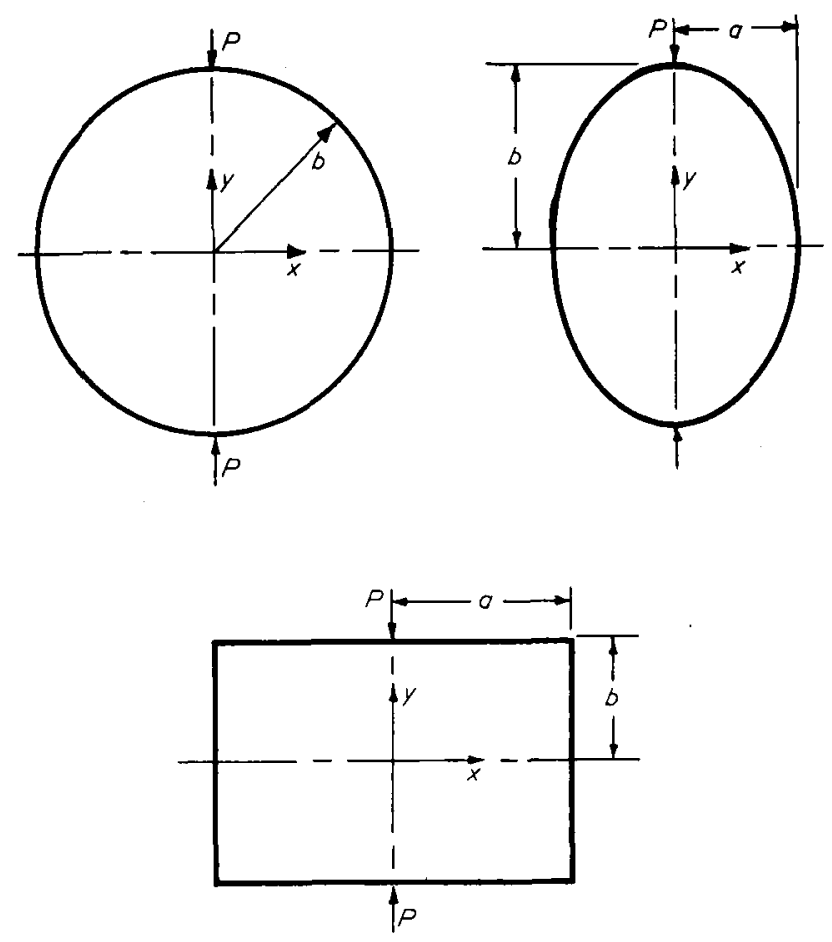

Depth of all members is one unit.

Fig. 2. Description and definitions for compression members considered

An analysis of elliptical discs subjected to point loads is made, the width/height ratio being used as a parameter. The co-ordinate system and geometrical parameters are defined in Fig. 2. 192 segments were used to define the boundary. The principal stresses at the origin of several elliptical discs were determined and are tabulated in Table 1. The results presented by Brisbane (6) are also shown in Table 1. Despite the inherent error incurred in reading Brisbane's results from a graph, the results are in excellent agreement, differences being less than 2 per cent at those points where direct comparison is possible. These results show that the tensile stress at the origin as a function of the width/height ratio is a maximum for a circular disc.

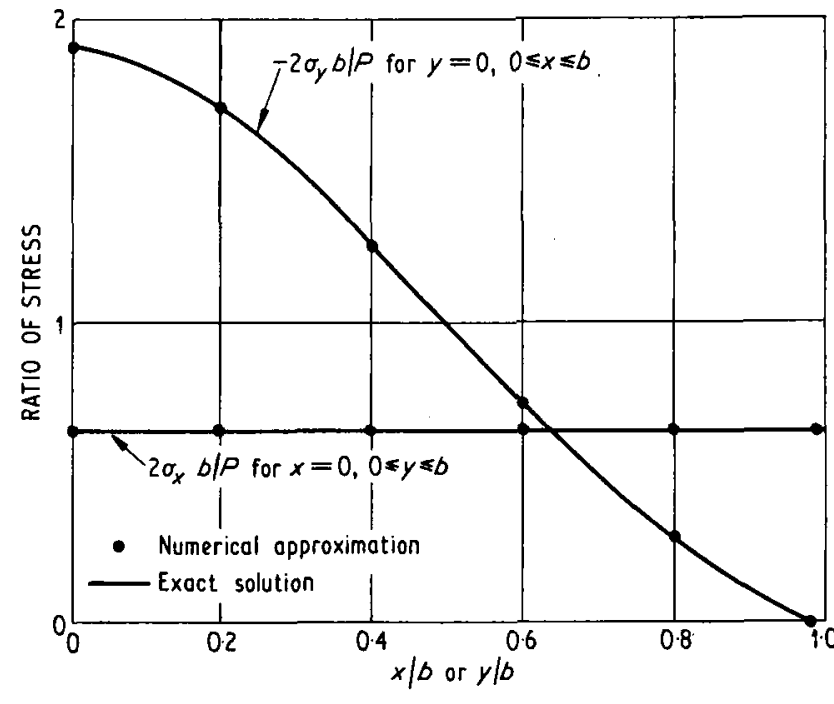

Fig. 3. Stress distributions in a circular disc subjected to diametrically opposed compressive point loads

While the principal stresses at the origin are of interest, the maximum value of the tensile stress $\sigma_{x}$ is found to occur in every case at $y= \pm b$ except the circular disc where the tensile stress is the same at all points along the $y$ axis. Tensile-stress distributions along the $y$ axis are shown in Fig. 4 for two elliptical discs and a circular disc. Fig. 5 and Table 2 show the maximum tensile stress in elliptical discs as a function of the width/height ratio. This parametric analysis indicates a relative minimum at $a / b$ equal to 1 (a circular disc) and reveals that as the width/height ratio approaches infinity, the maximum tensile stress approaches asymptotically the result for an infinite strip subjected to point loads as recorded in (3).

An analysis of rectangular blocks subjected to point loads is made, the width/height ratio of the block being used as a parameter. For the solutions 192 segments were used to describe the boundary. The solutions were relatively insensitive to the placement of boundary segments but some concentration of segments in the neighbourhood of the sharp corners was necessary to limit the "corner effect' that has been discussed by Massonnet (7). The coordinate system and geometrical parameters are defined in

Table 1. Comparison of principal stresses at the origins of elliptical discs subjected to point loads

\begin{tabular}{|c|c|c|c|c|c|c|}
\hline \multirow[t]{2}{*}{$\begin{array}{l}\text { Width/height ratio } \\
\text { a/b (see Fig. 2) }\end{array}$} & \multicolumn{3}{|c|}{ Tensile stress $\frac{\sigma_{x}}{P /(2 b)}$} & \multicolumn{3}{|c|}{ Compressive stress $\frac{-\sigma_{y}}{P /(2 b)}$} \\
\hline & Numerical* & Photoelastic ${ }^{\star}$ & $\begin{array}{c}\text { Present } \\
\text { calculations }\end{array}$ & Numerical ${ }^{\star}$ & Photoelastic ${ }^{\star}$ & $\begin{array}{l}\text { Present } \\
\text { calculations }\end{array}$ \\
\hline $\begin{array}{l}0.50 \\
0.6667 \\
0.75 \\
0.90 \\
1.00 \\
1.10 \\
1.25 \\
1.50 \\
1.75 \\
2.00 \\
3.00 \\
4.00 \\
\quad \infty\end{array}$ & $\begin{array}{r}0.34 \\
0.51 \\
\overline{-} \\
0.64 \\
\overline{-} \\
0 . \overline{58} \\
0 . \overline{53} \\
\overline{-} \\
0 . \overline{49 \ddagger}\end{array}$ & $\begin{array}{c}0.36 \\
0.54 \\
- \\
- \\
- \\
- \\
- \\
-\end{array}$ & $\begin{array}{l}0.345 \\
0.51 \dagger \\
0.574 \\
0.626 \\
0.632 \\
0.627 \\
0.608 \\
0.574 \\
0.549 \\
0.532 \\
0.502 \\
0.490 \\
-\end{array}$ & $\begin{array}{c}2.26 \\
1.95 \\
-\overline{1} \\
1.89 \\
\overline{-} \\
1.89 \\
\overline{1.87} \\
\overline{-} \\
1.825\end{array}$ & $\begin{array}{c}2 \cdot 26 \\
1.95 \\
- \\
- \\
- \\
- \\
- \\
- \\
-\end{array}$ & $\begin{array}{l}2.247 \\
2.00 \dagger \\
1.934 \\
1.913 \\
1.912 \\
1.911 \\
1.907 \\
1.894 \\
1.881 \\
1.872 \\
1.854 \\
1.848 \\
-\end{array}$ \\
\hline
\end{tabular}


F. J. APPL

Table 2. Location and magnitude of various levels of tensile stress on the $y$ axis of elliptical discs

\begin{tabular}{|c|c|c|c|c|c|c|c|c|}
\hline \multirow[t]{2}{*}{$\begin{array}{l}\text { Width/height ratio } \\
\qquad a / b\end{array}$} & \multicolumn{2}{|c|}{ Maximum tensile stress } & \multicolumn{2}{|c|}{$\begin{array}{l}95 \text { per cent of maximum } \\
\text { tensile stress }\end{array}$} & \multicolumn{2}{|c|}{$\begin{array}{l}90 \text { per cent of maximum } \\
\text { tensile stress }\end{array}$} & \multicolumn{2}{|c|}{$\begin{array}{c}80 \text { per cent of maximum } \\
\text { tensile stress }\end{array}$} \\
\hline & $\begin{array}{c}\text { Magnitude } \\
\frac{\sigma_{x}}{P / b}\end{array}$ & $\begin{array}{l}\text { Location } \\
\quad \pm y / b\end{array}$ & $\begin{array}{l}\text { Magnitude } \\
\frac{0.95 \sigma_{x}}{\bar{P} / b}\end{array}$ & $\begin{array}{l}\text { Location } \\
\pm y / b\end{array}$ & $\begin{array}{c}\text { Magnitude } \\
\frac{0 \cdot 90 \sigma_{x}}{P / b}\end{array}$ & $\begin{array}{l}\text { Location } \\
\pm y / b\end{array}$ & $\begin{array}{c}\text { Magnitude } \\
\frac{0 \cdot 80 \sigma_{x}}{P / b}\end{array}$ & $\begin{array}{l}\text { Location } \\
\quad \pm y / b\end{array}$ \\
\hline $\begin{array}{l}0.50 \\
0.75 \\
0.90 \\
1.00 \\
1.10 \\
1.25 \\
1.50 \\
1.75 \\
2.00 \\
3.00 \\
4.00\end{array}$ & $\begin{array}{l}0.726 \\
0.381 \\
0.326 \\
0.318 \\
0.323 \\
0.341 \\
0.375 \\
0.402 \\
0.420 \\
0.453 \\
0.464\end{array}$ & $\begin{array}{l}1.000 \\
1.000 \\
1.000 \\
t \\
1.000 \\
1.000 \\
1.000 \\
1.000 \\
1.000 \\
1.000 \\
1.000\end{array}$ & $\begin{array}{c}0.690 \\
0.362 \\
\star \\
\star \\
\star \\
0.324 \\
0.356 \\
0.381 \\
0.399 \\
0.430 \\
0.441\end{array}$ & $\begin{array}{l}0.980 \\
0.900 \\
\bar{Z} \\
\bar{Z} \\
0.738 \\
0.894 \\
0.925 \\
0.938 \\
0.953 \\
0.958\end{array}$ & $\begin{array}{c}0.654 \\
0.343 \\
\star \\
\star \\
\star \\
0.307 \\
0.338 \\
0.361 \\
0.378 \\
0.408 \\
0.418\end{array}$ & $\begin{array}{c}0.959 \\
0.788 \\
= \\
\overline{-} \\
0.250 \\
0.769 \\
0.844 \\
0.869 \\
0.903 \\
0.913\end{array}$ & $\begin{array}{c}0.581 \\
0.304 \\
\star \\
\star \\
\star \\
\star \\
0.300 \\
0.321 \\
0.336 \\
0.362 \\
0.371\end{array}$ & 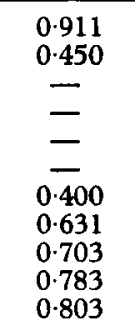 \\
\hline
\end{tabular}

- Tensile stress at all points on the $y$ axis is greater than the stress level indicated.

$\dagger$ Tensile stress is the same at all points on the $y$ axis.

Table 3. Location and magnitude of various levels of tensile stress on the $y$ axis of rectangular blocks

\begin{tabular}{|c|c|c|c|c|c|c|c|c|}
\hline \multirow[t]{2}{*}{$\begin{array}{l}\text { Width/height ratio } \\
a / b\end{array}$} & \multicolumn{2}{|c|}{ Maximum tensile stress } & \multicolumn{2}{|c|}{$\begin{array}{l}95 \text { per cent of maximum } \\
\text { tensile stress }\end{array}$} & \multicolumn{2}{|c|}{$\begin{array}{l}90 \text { per cent of maximum } \\
\text { tensile stress }\end{array}$} & \multicolumn{2}{|c|}{$\begin{array}{l}80 \text { per cent of maximum } \\
\text { tensile stress }\end{array}$} \\
\hline & $\begin{array}{c}\text { Magnitude } \\
\frac{\sigma_{x}}{P / b}\end{array}$ & $\begin{array}{l}\text { Location } \\
\quad \pm y / b\end{array}$ & $\begin{array}{c}\text { Magnitude } \\
\frac{0.95 \sigma_{x}}{P / b}\end{array}$ & $\begin{array}{l}\text { Location } \\
\pm y / b\end{array}$ & $\begin{array}{c}\text { Magnitude } \\
\frac{0.90 \sigma_{x}}{P / b}\end{array}$ & $\begin{array}{l}\text { Location } \\
\pm y / b\end{array}$ & $\begin{array}{c}\text { Magnitude } \\
\frac{0 \cdot 80 \sigma_{x}}{P / b}\end{array}$ & $\begin{array}{l}\text { Location } \\
\quad \pm y / b\end{array}$ \\
\hline $\begin{array}{l}0.25 \\
0.50 \\
0.75 \\
0.90 \\
1.00 \\
1.25 \\
1.50 \\
2.00 \\
3.00\end{array}$ & $\begin{array}{l}1.150 \\
0.577 \\
0.403 \\
0.387 \\
0.396 \\
0.437 \\
0.467 \\
0.485 \\
0.486\end{array}$ & $\begin{array}{l}1.000 \\
1.000 \\
1.000 \\
1.000 \\
1.000 \\
1.000 \\
1.000 \\
1.000 \\
1.000\end{array}$ & $\begin{array}{l}1.093 \\
0.548 \\
0.383 \\
0.367 \\
0.376 \\
0.415 \\
0.443 \\
0.461 \\
0.461\end{array}$ & $\begin{array}{l}0.991 \\
0.977 \\
0.964 \\
0.963 \\
0.963 \\
0.963 \\
0.963 \\
0.963 \\
0.963\end{array}$ & $\begin{array}{l}1.035 \\
0.519 \\
0.363 \\
0.348 \\
0.356 \\
0.393 \\
0.420 \\
0.436 \\
0.437\end{array}$ & $\begin{array}{l}0.979 \\
0.950 \\
0.920 \\
0.916 \\
0.916 \\
0.922 \\
0.922 \\
0.922 \\
0.920\end{array}$ & $\begin{array}{l}0.920 \\
0.462 \\
0.322 \\
0.309 \\
0.316 \\
0.349 \\
0.373 \\
0.388 \\
0.388\end{array}$ & $\begin{array}{l}0.940 \\
0.864 \\
0.738 \\
0.713 \\
0.763 \\
0.809 \\
0.822 \\
0.822 \\
0.822\end{array}$ \\
\hline
\end{tabular}

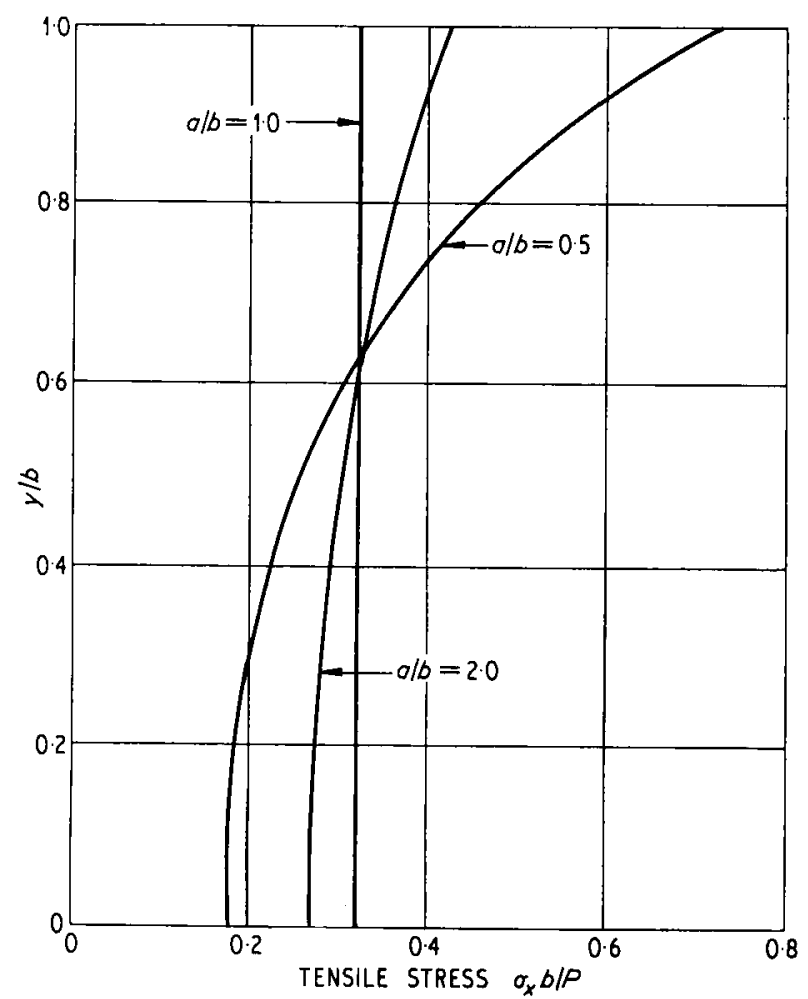

Fig. 4. Tensile-stress distributions on the $y$ axis in three elliptical discs subjected to diametrically opposed compressive point loads
Fig. 2. Tensile-stress distributions along the $y$ axis are shown in Fig. 6 for three specific width/height ratios. Fig. 5 and Table 3 show the maximum tensile stress in rectangular blocks as a function of the width/height ratio. As shown in Fig. 5, the maximum tensile stress in rectangular blocks as a function of $a / b$ assumes a relative minimum when $a / b=0.9$ and asymptotically approaches the limiting value for an infinitely wide block as $a / b$ becomes large.

The maximum tensile stresses are compared with those determined by Goodier (3) in Table 4. These tensile-stress magnitudes agree relatively well; a maximum difference of approximately 6 per cent is indicated for a square block. The tensile-stress distributions currently determined are very similar to those determined by Goodier (3) for $a / b=1$ and 2. However, the tensile-stress distribution shown in Fig. 6 for $a / b=0.5$ is quite different from that

Table 4. Comparison of maximum tensile stresses in rectangular blocks subjected to point loads

\begin{tabular}{c|c|c}
\hline $\begin{array}{c}\text { Width/height ratio } \\
a / b \text { (see Fig. 2) }\end{array}$ & \multicolumn{2}{|c}{ Maximum tensile stress $\frac{\sigma_{x}}{P / b}$} \\
& Goodier (3) & Present calculations \\
\hline 0.50 & 0.58 & 0.577 \\
1.00 & 0.42 & 0.396 \\
2.00 & 0.48 & 0.485 \\
\hline
\end{tabular}




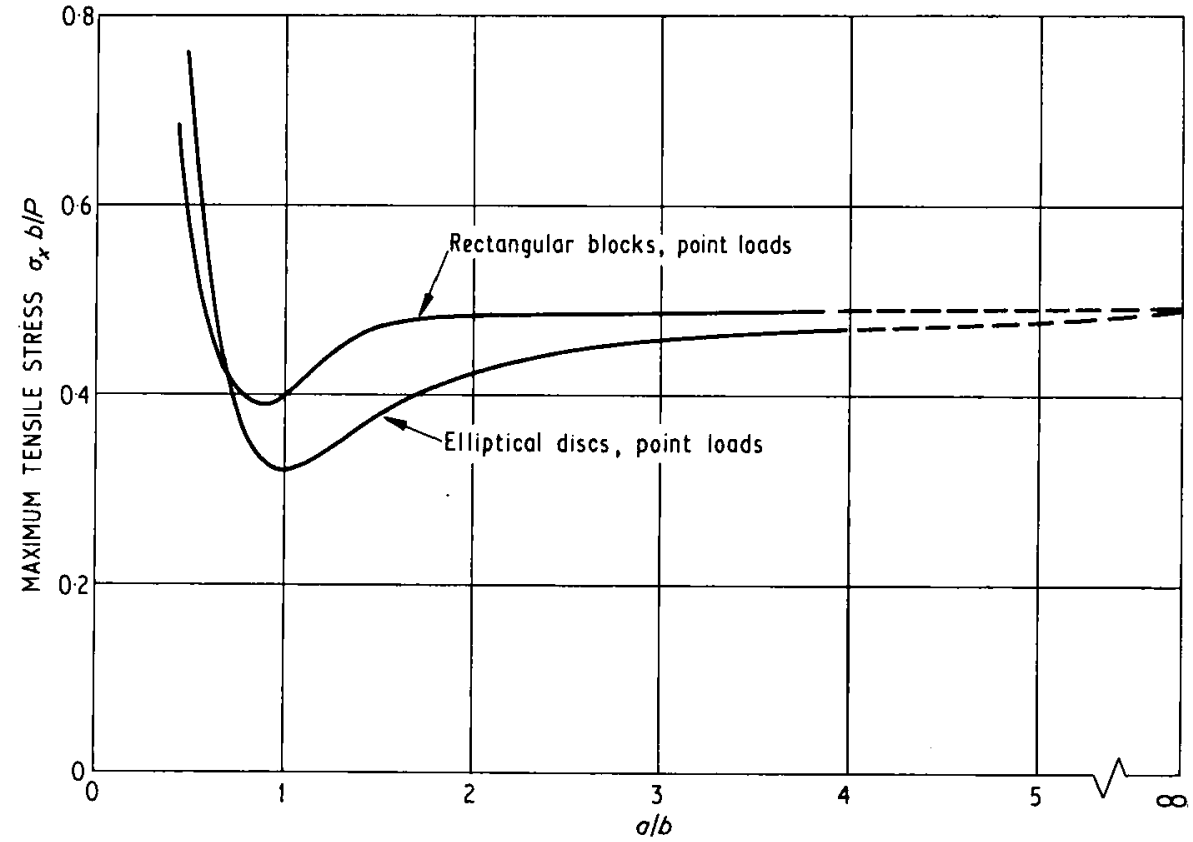

Fig. 5. Parametric analysis of maximum tensile stresses on the $y$ axis of compression members

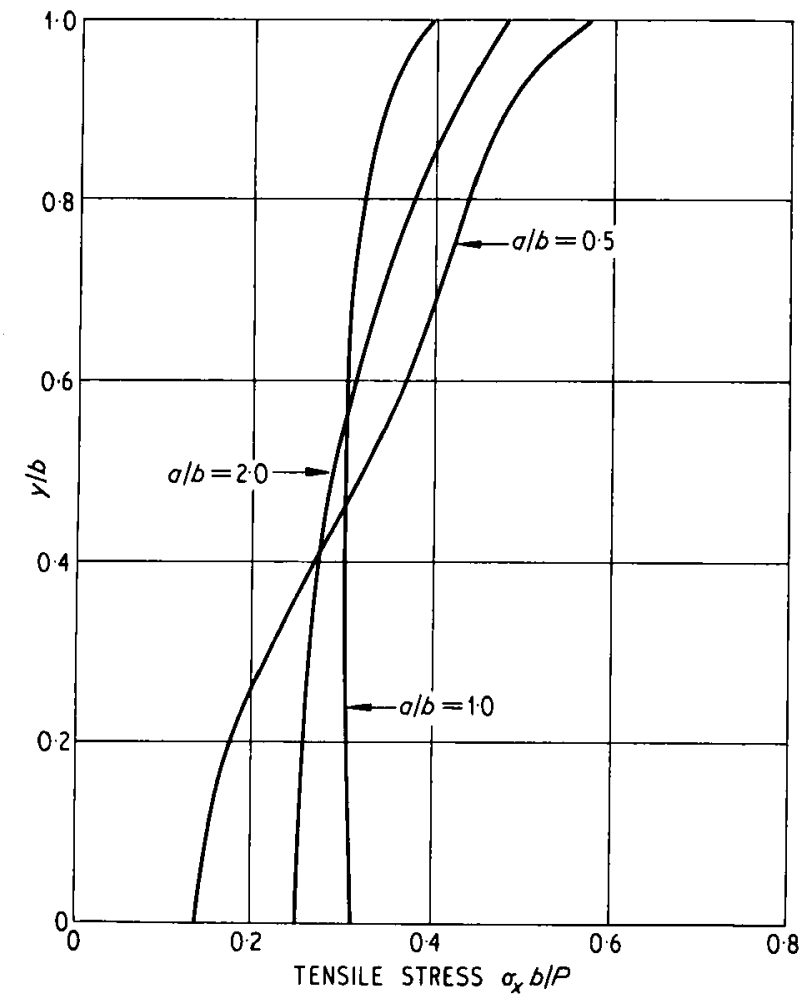

Fig. 6. Tensile-stress distributions on the $y$ axis in three rectangular blocks subjected to compressive point loads

in (3). The distribution of tensile stress in (3) shows a maximum value at $y / b=0.8$ and a lesser value at $y / b= \pm 1$. The present analysis indicates that the maximum tensile stress on the $y$ axis always occurs when $y / b= \pm 1$

Results of a photoelastic analysis of a rectangular block subjected to point loads have been published as a portion of a paper by Phillips and Mantei (9). The specific member investigated had a width/height ratio of 0.305 as referred to Fig. 2. Experimental data are shown for the tensile stress $\sigma_{x}$ acting on the line of action of the external loads, the $y$ axis. In the vicinity of the point loads the experimental data in (9) are incomplete and the curve showing tensile stress as a function of location along the $y$ axis represents an extrapolation which, as Phillips and Mantei (9) point out, is uncertain.

A comparison is shown in Fig. 7 between the experimental data as read from Fig. 4 of (9) and the results of a numerical analysis of this problem as determined by the method presented here. The agreement between experimental data and the numerical analysis is reasonably good

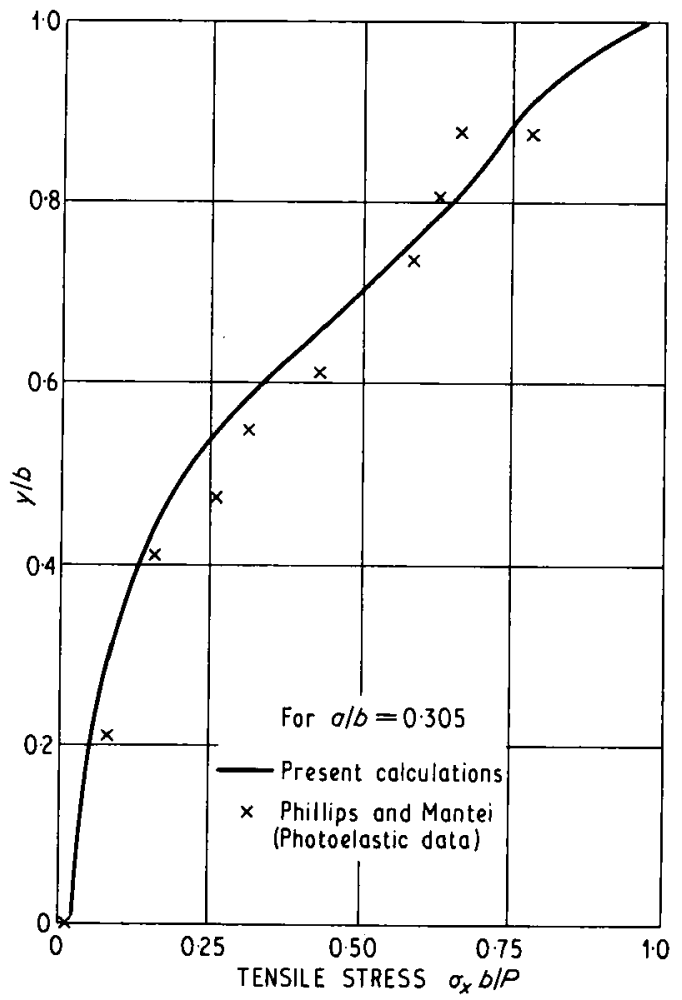

Fig. 7. Comparison of tensile stresses on the $y$ axis for a rectangular block with $a / b=0.305$ and subjected to compressive point loads 
Table 5. Comparison of stresses in a rectangular block with $a / b=2$ and subjected to point loads

\begin{tabular}{l|c|c|c}
\hline$y / b$ & $x / a$ (see Fig. 2) & \multicolumn{2}{|c}{ Tensile stress $\frac{\sigma_{x}}{P / b}$} \\
& & Gaydon (5) & $\begin{array}{c}\text { Present } \\
\text { calculations }\end{array}$ \\
& & & 0.249 \\
& & 0.251 & 0.255 \\
0.2 & 0 & 0.257 & 0.275 \\
0.4 & 0 & 0.278 & 0.313 \\
0.6 & 0 & 0.317 & 0.378 \\
0.8 & 0 & 0.382 & 0.425 \\
0.9 & 0 & - & 0.485 \\
1.0 & 0 & $\infty$ & $-\sigma_{y}$ \\
& & & Compressive stress $\frac{0}{P / b}$ \\
\hline 0 & 0 & 0.923 & 0.925 \\
0 & & $\infty$ & $\infty$ \\
\hline
\end{tabular}

throughout the range of the experimental data. The present calculations for this specific case indicate a distribution of tensile stress in the vicinity of the points of load application which is more consistent with the trend of the results in (3), (5), and (6) and of other cases presented herein than is the extrapolation shown in (9).

Table 5 gives a comparison of the solutions for a block with $a / b=2.0$ as determined in (5) and in the present work. With the exception of one point, the solutions differ by 1 per cent or less. At the point $x / a=0$ and $y / b=$ 1.0 , Gaydon indicates a tensile stress of infinity, while the present calculations indicate a tensile stress of 0.485 which is consistent with an asymptotic approach to the solution for an infinitely wide block (5) as shown in Fig. 5.

The redistribution of stress according to Saint-Venant's principle is shown in Fig. 8 for the rectangular block with $a / b=0.5$ and subjected to point loads. At a distance equal to one width away from the point load, the compressivestress distribution is found to differ from a uniform stress distribution by 6.7 per cent.

Some materials are 'flaw sensitive'. For an accurate determination of the fracture strength of this type of material it is imperative that a relatively large volume of material be subjected to a high level of tensile stress. Tables 2 and 3 show the location along the $y$ axis of the 95 per cent level of maximum tensile stress, the 90 per cent level, and the 80 per cent level for various elliptical discs and rectangular blocks. Since the maximum tensile stresses in these members occur when $y / b= \pm 1 \cdot 0$, the values of $y / b$ given for the various stress levels indicate the percentage of the $y$ axis subjected to tensile stresses less than the level indicated. For example, Table 3 shows that in the square block 3.7 per cent of the $y$ axis is subjected to a tensile stress of 95 per cent or more of the maximum tensile stress, 8.4 per cent is subjected to a tensile stress of 90 per cent or more of the maximum, and 23.7 per cent is subjected to a tensile stress of 80 per cent or more of the maximum. The distribution in the square block may be compared with the distribution in ellipses for which $0.9 \leqslant a / b \leqslant 1.1$, where the entire $y$ axis is subjected to tensile stresses greater than 95 per cent of the maximum tensile stress.

While these stress distributions along the $y$ axis are not sufficient to determine absolute volumes of highly stressed

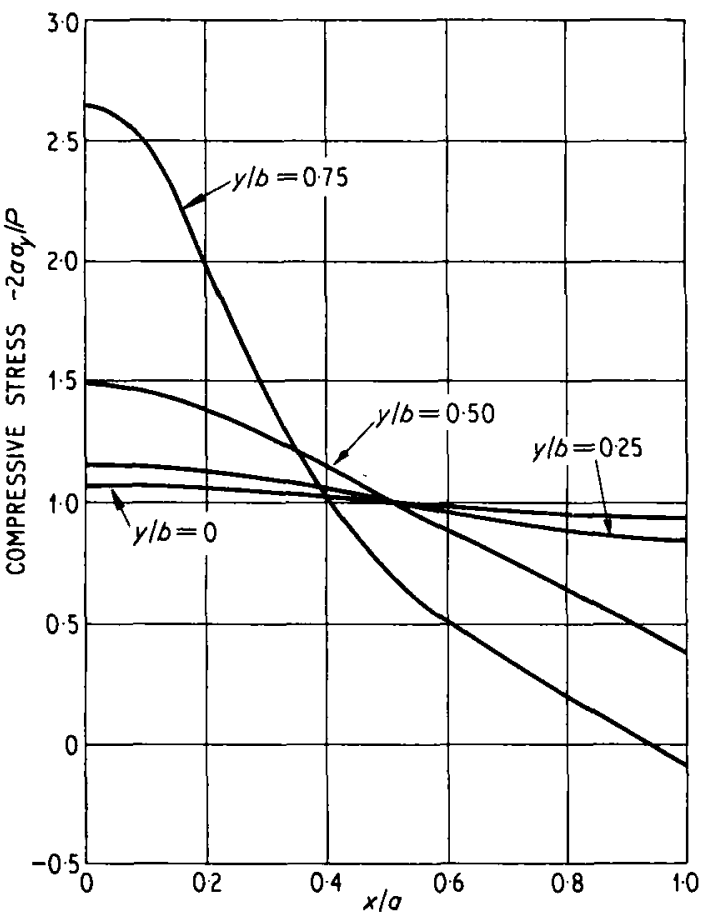

Fig. 8. Compressive-stress distributions in a rectangular block with $a / b=0.5$ and subjected to compressive point loads

material, they do give an indication as to which geometrical shapes are best for experimental tests with 'flaw sensitive' material. It is apparent from Tables 2 and 3 that only the elliptical discs with $0.9 \leqslant a / b \leqslant 1 \cdot 1$ have the entire $y$ axis subjected to 95 per cent or more of the maximum stress.

While the elliptical discs in the range of $0.9 \leqslant a / b \leqslant 1 \cdot 1$ have the most uniform tensile-stress distributions along the $y$ axis, they also exhibit the lowest magnitudes of maximum tensile stress as shown in Tables 2 and 3. If it is desired to produce the greatest values of tensile stress for a given magnitude of applied load, it is apparent that the circular disc is not a good choice. It may also be observed that generally the maximum tensile stresses change very rapidly for values of $a / b$ less than $1 \cdot 0$. Accurate correlation between applied load and maximum tensile stress will thus be highly dependent on an accurate determination of the parameter $a / b$ for $a / b$ less than $1 \cdot 0$.

\section{CONCLUSIONS}

The superposition method developed in this paper is applicable to plane elasticity problems that have concentrated forces acting on the boundaries. The solutions are equally valid for plane stress or plane strain. In the superposition method, the effects of concentrated forces (point loads) are represented exactly by use of Flamant solutions and a singular integral method of numerical analysis is used to satisfy the boundary conditions at discrete points on the remainder of the boundary. The accuracy of the method is illustrated by comparing the numerical analysis with the exact solution for a circular disc subjected to diametrically opposed point loads and with previous solutions for specific cases. The versatility of the method is indicated by the range of problems considered.

Parametric analyses of rectangular blocks and elliptical discs subjected to point loads as functions of the width/ 
height ratio are presented. The results compare well with solutions of specific cases previously investigated. With the results of these parametric analyses, an accuracte correlation between external compressive loads and the maximum tensile stress in variously proportioned members is possible. With these correlations, the relation between external compressive load and tensile fracture strength of brittle materials is available for use in analysing the results of indirect testing of members of various shapes. An indication of the relative volume of material that is subjected to high tensile stresses is presented as a function of the width/height ratio of the various members for use in designing members used to determine the fracture strength of brittle materials that are 'flaw sensitive'.

For brittle materials that are 'flaw sensitive', an elliptical disc with a width/height ratio between 0.9 and 1.1 should be used to ensure that the entire cross-section is subjected to tensile stresses which are greater than 95 per cent of the maximum tensile stress. For brittle materials that are not particularly 'flaw sensitive', it may be desirable to produce the greatest possible tensile stress for a given applied load. Greater maximum tensile stresses for a given applied load can be produced in either elliptical or rectangular members which have width/height ratios either considerably less or greater than $1 \cdot 0$.

\section{ACKNOWLEDGEMENTS}

The work reported herein was initiated in the Research Department of the Caterpillar Tractor Company, Peoria, IIl., and was completed at The University of Oklahoma, Norman, Oklahoma.

\section{APPENDIX}

REFERENCES

(I) Timoshenko, S. and GoodieR, J. N. Theory of elasticity 3rd edition 1970 (McGraw-Hill Book Co. Inc., New York and Maidenhead).

(2) APPL, F. J. and KoERNER, D. R. 'Numerical analysis of plane eleasticity problems', 7. Engng Mech. Div. Am. Soc. civ. Engrs 196894 (No. EM3), 743-752.

(3) Goodier, J. N. 'Compression of rectangular blocks, and the bending of beams by non-linear distributions of bending forces', Trans. Am. Soc. mech. Engrs 1932 54, APM 173-183.

(4) Niedenfuhr, F. W., Leissa, A. W., and Lo, C. C. 'A study of the point matching method as applied to thermally and transversely loaded plates and other boundary value problems', $A F F D L-T R-64-159$, Air Force Flight Dynamics Laboratory, Wright-Patterson Air Force Base, Ohio, 1964.

(5) GAyDon, F. A. 'The rectangle in generalized plane stress, with numerically prescribed boundary stresses and applications to discontinuous loading', Proc. $R$. Soc. Series A 1965 286, 251-269.

(6) Brisbane, J. J. 'Axial loading of an elliptical disk', f. appl. Mech., Trans. Am. Soc. mech. Engrs 1963 85, 306-309.

(7) Massonnet, C. E. 'Numerical use of integral procedures', Stress analysis (edited by Zienkiewicz, $\mathrm{O}$. C. and Holister, G. S.) 1965 198-235 (John Wiley and Sons Inc., New York and London).

(8) Lo, C. C., NiedenfuHR, F. W., and Leissa, A. W. 'Further studies in the application of the point matching technique to plate bending and other harmonic and biharmonic boundary value problems', $A F F D L-T R-65-114$, Air Force Flight Dynamics Laboratory, Wright-Patterson Air Force Base, Ohio, 1966.

(9) Phillips, H. B. and Mantei, C. L. 'Two-dimensional study of transverse stresses in axially loaded sections', Expl Mech. 1969 9, 137-139. 